\title{
Avaliação da influência de comorbidades cardiometabólicas sobre a evolução clínica da covid-19 em pacientes com lúpus eritematoso sistêmico no Amazonas
}

\author{
Evaluation of the influence of cardiometabolic comorbidities on the clinical evolution of \\ covid-19 in patients with systemic lupus erythematosus in Amazonas \\ Evaluación de la influencia de las comorbilidades cardiometabólicas en la evolución \\ clínica de COVID-19 en pacientes con lupus eritematoso sistémico en Amazonas
}

Andrezza Mendes Franco ${ }^{1 *}$, Sebastião Barreto Falcão Neto ${ }^{1}$, Camile Smith de Oliveira Brito Rosana Barros de Souza', Sandra Lúcia Euzébio Ribeiro'.

\section{RESUMO}

Objetivo: Avaliar a influência de comorbidades cardiometabólicas sobre a evolução da Doença de Coronavírus de 2019 (COVID-19) nos portadores de Lúpus Eritematoso Sistêmico (LES) no Amazonas. Métodos: Estudo prospectivo, observacional e descritivo, constituinte do projeto guarda-chuva REUMACOV BRASIL. Avaliou-se as fichas dos pacientes com LES do projeto REUMACOV no Amazonas e coletados dados sociodemográficos, presença de comorbidades e evolução clínica da COVID-19. Resultados: Amostra com 137 pacientes com LES, sendo 76 com COVID-19 (grupo Caso) e 61 saudáveis (grupo Controle). Havia comorbidades em $56,4 \%$ do Caso e $43,6 \%$ do Controle, as principais foram Hipertensão Arterial e Obesidade. O principal achado laboratorial foi a elevação de marcadores inflamatórios no Caso, especialmente naqueles com doenças associadas. Maiores taxas de hospitalizações, óbitos e limitações pós-COVID-19 ocorreram nos com comorbidades, mesmo sem relevância estatística. Além disso, o período sintomático foi maior nesse grupo. Conclusão: A influência das comorbidades sobre o prognóstico de pacientes com LES e COVID-19 foi pequena, pois embora dados tenham mostrado que esse grupo foi mais suscetível a quadros preocupantes, estatisticamente ele se mostrou semelhante ao grupo sem comorbidades.

Palavras-chave: COVID-19, Lúpus eritematoso sistêmico, Hipertensão arterial sistêmica, Diabetes mellitus, Obesidade.

\begin{abstract}
Objective: To evaluate the influence of cardiometabolic comorbidities on the evolution of Coronavirus Disease 2019 (COVID-19) in patients with Systemic Lupus Erythematosus (SLE) in Amazonas. Methods: Prospective, observational and descriptive study, part of the umbrella project REUMACOV BRASIL. The records of patients with SLE from the REUMACOV project in Amazonas were evaluated and sociodemographic data, presence of comorbidities and clinical evolution of COVID-19 were collected. Results: Sample with 137 SLE patients, 76 with COVID-19 (Case group) and 61 healthy (Control group). There were comorbidities in $56.4 \%$ of the Case and $43.6 \%$ of the Control, the main ones being Arterial Hypertension and Obesity. The main laboratory finding was the elevation of inflammatory markers in the case, especially in those with associated diseases. Higher rates of hospitalizations, deaths and post-COVID-19 limitations occurred in those with comorbidities, even without statistical significance. Furthermore, the symptomatic period was longer in this group. Conclusion: The influence of comorbidities on the prognosis of patients with SLE and COVID-19 was small, as although data have shown that this group was more susceptible to worrying conditions, it was statistically similar to the group without comorbidities.
\end{abstract}

Keywords: COVID-19, Systemic lupus erythematosus, Systemic arterial hypertension, Diabetes mellitus, Obesity.

1 Universidade Federal do Amazonas (UFAM), Manaus - AM. *E-mail: andrezzamf2190@gmail.com

O estudo faz parte do Programa de Iniciação Científica (PIBIC) da Universidade Federal do Amazonas (UFAM), 2020-2021, com bolsa pela CNPq. 


\section{RESUMEN}

Objetivo: Evaluar la influencia de las comorbilidades cardiometabólicas en la evolución de la Enfermedad por Coronavirus 2019 (COVID-19) en pacientes con Lupus Eritematoso Sistémico (LES) en Amazonas. Métodos: Estudio prospectivo, observacional y descriptivo, que forma parte del proyecto paraguas REUMACOV BRASIL. Se evaluaron los registros de pacientes con LES del proyecto REUMACOV en Amazonas y se recolectaron datos sociodemográficos, presencia de comorbilidades y evolución clínica de COVID-19. Resultados: Muestra con 137 pacientes con LES, 76 con COVID-19 (grupo Caso) y 61 sanos (grupo Control). Hubo comorbilidades en el 56,4\% del Caso y el $43,6 \%$ del Control, siendo las principales Hipertensión Arterial y Obesidad. El principal hallazgo de laboratorio fue la elevación de marcadores inflamatorios en el caso, especialmente en aquellos con enfermedades asociadas. Las tasas más altas de hospitalizaciones, muertes y limitaciones post-COVID-19 ocurrieron en aquellos con comorbilidades, incluso sin significación estadística. Además, el período sintomático fue más largo en este grupo. Conclusión: La influencia de las comorbilidades en el pronóstico de los pacientes con LES y COVID-19 fue pequeña, ya que aunque los datos han demostrado que este grupo era más susceptible a condiciones preocupantes, fue estadísticamente similar al grupo sin comorbilidades.

Palabras clave: COVID-19, Lupus eritematoso sistémico, Hipertensión arterial sistémica, Diabetes mellitus, Obesidad.

\section{INTRODUÇÃO}

A Doença de Coronavírus de 2019 (COVID-19) surgiu em dezembro de 2019 na cidade de Wuhan na China e atingiu proporções pandêmicas em poucos meses, tornando-se uma emergência de saúde pública (MUNIYAPPA R e GUBBI S, 2020). Em 16 de Agosto de 2021, segundo o Mapa da COVID-19 do Johns Hopkins Coronavirus Resource Center (2021), o número de casos confirmados de COVID-19 em todo o mundo era de 207.422.686 milhões e o número de mortes de 4.366.259 milhões. No Brasil, esses números são de 20.364.099 milhões e 569.058 mil, respectivamente.

A COVID-19 é uma infecção viral que tem como principais sintomas febre, tosse sem expectoração, dispnéia, astenia, cefaléia, dor de garganta, rinorreia, espirros, náuseas, vômitos, diarréia e quadros de linfopenia que pode ter complicações como a Síndrome Respiratória Aguda Grave (SRAG) e a Síndrome de Tempestade de Citocinas, podendo levar ao óbito(ROTHAN HA e BYRAREDDY SN, 2020). A transmissão é feita pelo contato pessoa-pessoa por meio de gotículas respiratórias ou contato com objetos contaminados (BAl Y et al., 2020).

Atualmente existem cerca de 10 vacinas aprovadas no mundo contra a COVID-19, sendo que 4 delas estão sendo aplicadas no Brasil. Aproximadamente $31,4 \%$ da população mundial foi vacinada com a primeira dose ou dose única, e outros $23,6 \%$ estão completamente imunizados (ROSER H, 2021). Segundo o Ministério da Saúde (2021), até 16 de Agosto de 2021 foram aplicadas um total de 164.638.573 milhões de doses, incluindo dose única, primeira e segunda dose.

O agente etiológico é o vírus da Síndrome Respiratória Aguda Grave do Coronavírus 2 (SARS-CoV-2), um vírus de RNA envelopado de cadeia única, classificado como um beta coronavírus e o sétimo de sua família, de origem zoonótica (ZHU N et al., 2020).

A fisiopatologia da infecção pelo SARS-CoV-2 é mediada pela ligação da proteína Spike (S) com os receptores da Enzima Conversora de Angiotensina 2 (ECA 2), o que vai promover a internalização do vírus. O receptor da ECA 2 está presente nos pulmões, endotélio, coração, rins, pâncreas, tecido adiposo, entre outros e isso pode ser um fator que determina o tropismo do SARS-CoV 2 por esses órgãos. Após a ligação da proteína Spike com o receptor da ACE 2, a proteína TMPRSS2 é responsável pela clivagem da proteína $S$ e ativação dos mecanismos de replicação do vírus (ASKIN L, et al., 2020).

As células infectadas pelo vírus sofrem processos de degeneração celular que desencadeiam cascatas inflamatórias com a participação de citocinas, quimiocinas, linfócitos TCD4+ auxiliares, linfócitos TCD8, entre outros que culminam em quadros de linfopenia e eventualmente na chamada "tempestade de citocinas"(MEHTA P, et al., 2020). 
Os grupos de pacientes que estão mais propensos a desfechos desfavoráveis da infecção pelo SARSCoV-2 são aqueles que possuem idade mais avançada, sexo masculino, tabagistas e portadores de comorbidades, como a Hipertensão Arterial Sistêmica (HAS), Diabetes Mellitus (DM), Cardiopatias, Pneumopatias e Imunossuprimidos. Além disso, achados laboratoriais de leucopenia, linfopenia, aumento de D-dímero, Proteína C Reativa (PCR), Desidrogenase Lática (DHL) e de biomarcadores cardíacos podem estar presents (ZHOU F, et al., 2020).

Diante desse panorama, desponta a apreensão acerca do impacto do COVID-19 nas doenças reumáticas, dentre elas o Lúpus Eritematoso Sistêmico (LES). Tal preocupação é justificada pelo fato de que esses pacientes na maioria dos casos utilizam tratamentos imunossupressores e por isso apresentam maior risco quando contraem doenças infecciosas. Por outro lado, o uso desses medicamentos poderia conferir-Ihes uma vantagem ao amenizar as respostas imunes desreguladas que ocorrem em quadros graves de COVID-19 envolvendo a "Tempestade de Citocinas"(CERIBELLI A, et al., 2020).

O LES é uma doença autoimune sistêmica que tem uma incidência de 50,8/ 100.000 pessoas, afetando especialmente mulheres e a etnia afro-americana (O'NEILL S e CERVERA R, 2010; KENJI O e TATSUYA A, 2018). O espectro sintomatológico do LES inclui desde manifestações cutâneas até acometimento renal, hematológico e neurológico, que evoluem em alternância de períodos de atividade e remissão da doença, sendo fundamental um acompanhamento regular para evitar complicações do quadro (PETRI M, et al., 2012). Uma série de estudos estão sendo feitos recentemente visando elucidar a relação LES e COVID-19, todavia os resultados preliminares são controversos (EMMI G, et al., 2020).

Desse modo, evidencia-se a necessidade de investigar melhor a influência do LES no curso da infecção pelo SARS-CoV-2, com enfoque na associação do LES com outras comorbidades e fatores de risco que comprovadamente desencadeiam apresentações clínicas mais graves e que ameaçam a vida dos pacientes. A partir disso, será possível fornecer dados que auxiliem no manejo clínico desses pacientes e no planejamento de ações de saúde pública diante do contexto pandêmico atual no Amazonas, um dos epicentros da COVID-19 no Brasil. Portanto, o objetivo deste estudo foi verificar o grau de influência de comorbidades sobre o curso da infecção pelo SARS-CoV-2 nos portadores de Lúpus Eritematoso Sistêmico (LES) atendidos por serviço de referência em Reumatologia no Amazonas.

\section{MÉTODOS}

Trata-se de um estudo de prospectivo, observacional e descritivo, conduzido como parte do projeto guarda-chuva "Estudo Brasileiro de Pacientes com Doenças Inflamatórias Crônicas Imunomediadas Infectados pelo Novo Coronavírus (SARS-CoV-2) - REUMACOV BRASIL", aprovado pelo Comitê de Ética em Pesquisa (CEP) da Universidade Federal do Amazonas (UFAM), com nCAAE 30186820.2.2016.5020 e no de parecer: 4.068.202.

Foi realizada uma análise com grupo de comparação e amostragem de conveniência, de adultos portadores de LES que se são casos suspeitos e/ou confirmados de COVID-19, além de pacientes controles que não contraíram o vírus. Inicialmente, com uma avaliação transversal sobre a sintomatologia prévia e/ou atual compatível com infecção e aspectos clínicos no momento da inclusão no estudo (presencialmente ou por via telefônica); e posteriormente o acompanhamento da evolução do caso por seis meses após a infecção viral.

Foram incluídos na pesquisa indivíduos de ambos os sexos com idade igual ou superior a 18 anos que possuíam diagnóstico de LES Segundo as diretrizes do Colégio Americano de Reumatologia (ACR) e da Liga Europeia Contra o Reumatismo (EULAR) de 2019. Além disso, aqueles com suspeita ou confirmação de COVID-19, de acordo com os protocolos do MS, foram alocados no grupo Caso, enquanto os demais no grupo Controle. Todos os participantes assinaram TCLE.

Excluíram-se do estudo pacientes considerados imunossuprimidos por outras causas, por exemplo infecção pelo vírus do HIV, transplante de órgãos, imunodeficiência primária, neoplasias, história de doenças do Timo (Miastenia Gravis, Timoma, Casos de Ausência do Timo ou Remoção Cirúrgica). 
A coleta de dados ocorreu no período de agosto de 2020 a Julho de 2021, por meio da verificação das fichas clínicas de cadastro e acompanhamento dos pacientes incluídos no projeto REUMACOV no Amazonas, obtidos junto à equipe do Serviço de Reumatologia do Ambulatório Araújo Lima, vinculado à Universidade Federal do Amazonas (UFAM). Os dados foram analisados individualmente e registrados em formulário online.

As variáveis examinadas incluem: № de identificação (um código utilizado para identificar cada paciente, visando preservar o anonimato); Data de Nascimento; Idade; Cidade; Sexo; Cor da Pele; Presença de Comorbidades, isto é, de Hipertensão Arterial Sistêmica, Cardiopatia, Diabetes Mellitus ou Obesidade. Sobre a COVID-19, caso houvesse diagnóstico da infecção e caso positivo, prosseguiu-se com o preenchimento das seguintes informações: Forma de contágio; Data de início dos sintomas; Duração dos sintomas; Quais foram os sintomas apresentados; Método de Diagnóstico; Achados laboratoriais; e Desfechos Clínicos do COVID-19.

Os resultados serão colocados em tabelas e gráficos e analisados através de estatística descritiva (incluindo frequência absoluta e relativa, média com desvio-padrão), teste qui-quadrado e exato de Fisher. Neste estudo, foram considerados como valores de significância, um $p$-valor $<0,05$.

\section{RESULTADOS}

\section{Descrição da amostra}

Participaram da pesquisa 137 pacientes diagnosticados com LES. A média de idade desse grupo foi de $38,9 \pm 10,4$ anos. Cerca de 125 (91,2\%) dos indivíduos eram do sexo feminino e 12(8,7\%) do masculino. A cor de pele predominante neste grupo foi de a Parda $(71,6 \%)$, seguida de Branca $(20,4 \%)$, Preta $(5,1 \%)$ e Amarela $(2,9 \%)$.

Acerca da prevalência de doenças concomitantes, no âmbito das patologias com impacto metabólico e cardiológico, foi constatado que 94 dos 137 pacientes tinham alguma comorbidade, havendo indivíduos com mais de uma comorbidade simultaneamente. A HAS foi a enfermidade com maior prevalência, acometendo $49,6 \%$ dos participantes, seguida da Obesidade (38,7\%), Dislipidemia (16,8\%), Diabetes Mellitus (10,2\%) e Cardiopatia $(3,7 \%)$.

\section{Avaliação dos pacientes com LES com e sem COVID-19}

Dentre os 137 participantes, 76 tinham com diagnóstico de COVID-19, segundo o protocolo do MS. Diante disso, a amostra foi dividida em 2 grupos: Grupo Caso, contendo os 76 pacientes com COVID-19; e o Grupo Controle, com 61 pacientes com LES que não se infectaram pelo SARS-COV-2. Na Tabela 1 estão apresentadas as características epidemiológicas dos pacientes de ambos os grupos.

Tabela 1 - Características Epidemiológicas dos pacientes pertencentes aos Grupos Caso e Controle ( $\mathrm{n}=137$ ).

\begin{tabular}{|c|c|c|c|c|c|c|}
\hline Variável & $\begin{array}{c}\text { Descrição da } \\
\text { variável }\end{array}$ & \multicolumn{2}{|c|}{$\begin{array}{c}\text { Grupo Caso } \\
N=76(55,5 \%)\end{array}$} & \multicolumn{2}{|c|}{$\begin{array}{c}\text { Grupo Controle } \\
\mathrm{N}=61(44,5 \%)\end{array}$} & P-valor \\
\hline Idade & $\begin{array}{l}\text { Idade em anos } \\
\text { - media (DP) }\end{array}$ & \multicolumn{2}{|c|}{$38 \pm 10,1$} & \multicolumn{2}{|c|}{$40,1 \pm 10,8$} & 0,120 \\
\hline Variável & $\begin{array}{l}\text { Descrição da } \\
\text { variável }\end{array}$ & $\begin{array}{c}\text { Frequência } \\
\text { absoluta } \\
\text { (n) }\end{array}$ & $\begin{array}{c}\text { Frequência } \\
\text { relativa } \\
(\%)\end{array}$ & $\begin{array}{c}\text { Frequência } \\
\text { absoluta } \\
\text { (n) }\end{array}$ & $\begin{array}{c}\text { Frequência } \\
\text { relativa } \\
(\%)\end{array}$ & P-valor \\
\hline Sexo & $\begin{array}{l}\text { Feminino } \\
\text { Masculino }\end{array}$ & $\begin{array}{c}69 \\
7\end{array}$ & $\begin{array}{c}90,8 \% \\
9,2 \%\end{array}$ & $\begin{array}{c}56 \\
5\end{array}$ & $\begin{array}{c}91,8 \% \\
8,2 \%\end{array}$ & 0,830 \\
\hline Cor da pele & $\begin{array}{c}\text { Branca } \\
\text { Parda } \\
\text { Preta } \\
\text { Amarela }\end{array}$ & $\begin{array}{c}13 \\
55 \\
6 \\
2\end{array}$ & $\begin{array}{c}17,1 \% \\
72,4 \% \\
7,9 \% \\
2,6 \%\end{array}$ & $\begin{array}{c}15 \\
43 \\
1 \\
2\end{array}$ & $\begin{array}{c}24,6 \% \\
70,5 \% \\
1,6 \% \\
3,3 \%\end{array}$ & 0,309 \\
\hline
\end{tabular}

Fonte: Franco AM, et al., 2021.

Sobre o método de diagnóstico, a maioria dos pacientes foi diagnosticada pelo critério clínicoepidemiológico 58(76,32\%); e apenas 18 fizeram exame laboratorial, sendo 10(13,16\%) Teste rápido, 
$3(3,95 \%)$ RT-PCR viral e $4(5,26 \%)$ sorologia IgM e IgG. Somente 4 pacientes realizaram Tomografia Computadorizada com alterações pulmonares ao momento do diagnóstico. Dos 76 pacientes, 27(35,53\%) referiram contato com caso suspeito/confirmado de COVID-19.

A maioria dos pacientes incluídos no estudo tiveram o diagnóstico de COVID-19 no mês de Abril/2020, haja vista que a inclusão de pacientes do grupo caso se deu principalmente neste período, não se refletindo nesses resultados a segunda onda de infecção pelo SARS-COV-2 registrada nos primeiros meses de 2021 no Amazonas.

Cerca de $53(56,4 \%)$ do grupo Caso e $41(43,6 \%)$ do grupo Controle apresentavam alguma comorbidade cardiometabólica. As mais frequentes foram HAS e a Obesidade, seguidas de DM, Dislipidemia e Cardiopatia. Encontrou-se diferenças significativas nas frequências de HAS, que foi mais observada no grupo Controle ( $p=0,049)$, observado na Tabela 2.

Tabela 2 - Prevalência de Comorbidades Cardiometabólicas nos pacientes estudados ( $n=137)$.

\begin{tabular}{cccccc}
\hline \multirow{2}{*}{ Variável } & \multicolumn{2}{c}{$\begin{array}{c}\text { Grupo Caso } \\
\mathbf{N}=\mathbf{7 6}(\mathbf{5 5 , 5 \% )}\end{array}$} & \multicolumn{2}{c}{$\begin{array}{c}\text { Grupo Controle } \\
\mathbf{N}=\mathbf{6 1}(\mathbf{4 4 , 5 \% )}\end{array}$} & \multirow{2}{*}{ P-valor } \\
\cline { 2 - 5 } & $\begin{array}{c}\text { Frequência } \\
\text { absoluta (n) }\end{array}$ & $\begin{array}{c}\text { Frequência } \\
\text { relativa (\%) }\end{array}$ & $\begin{array}{c}\text { Frequência } \\
\text { absoluta (n) }\end{array}$ & $\begin{array}{c}\text { Frequência } \\
\text { relativa (\%) }\end{array}$ & \\
\hline $\begin{array}{c}\text { Presença de } \\
\text { comorbidades }\end{array}$ & 53 & $56,4 \%$ & 41 & $43,6 \%$ & 0,752 \\
\hline $\begin{array}{c}\text { Hipertensão } \\
\text { arterial }\end{array}$ & 32 & $42,1 \%$ & 36 & $59,02 \%$ & 0,049 \\
\hline $\begin{array}{c}\text { Diabetes Mellitus } \\
\text { tipo 2 }\end{array}$ & 9 & $11,8 \%$ & 5 & $8,2 \%$ & 0,483 \\
\hline Obesidade & 33 & $42,4 \%$ & 20 & $32,8 \%$ & 0,204 \\
\hline Dislipidemia & 13 & $17,1 \%$ & 10 & $16,4 \%$ & 0,912 \\
\hline Cardiopatia & 4 & $5,3 \%$ & 1 & $1,64 \%$ & 0,261 \\
\hline
\end{tabular}

Fonte: Franco AM, et al., 2021.

Verificamos ainda os valores de Índice de Massa Corporal (IMC) e circunferência abdominal nos pacientes destes grupos. O IMC é um dos instrumentos utilizados para diagnosticar obesidade. A comparação entre o grupo caso e controle revelou uma tendência de os pacientes casos terem maiores índices de sobrepeso e obesidade em relação aos controles $(p=0,078)$. A circunferência abdominal aumentada, por sua vez, é um fator de risco para Síndrome Metabólica e ocorrência de eventos cardiovasculares. Na avaliação desta variável, $53,9 \%$ do grupo caso tinha um valor de circunferência acima dos padrões recomendados pela OMS, e no controle $44,26 \%$, não havendo diferença significativa entre os grupos.

Quanto aos achados laboratoriais, poucos pacientes fizeram seus exames e apenas alguns realizaram todos os exames solicitados no estudo, por dificuldade de realização devido a pandemia de COVID-19. No comparativo entre os grupos Caso e Controle, no grupo Caso, 60 pacientes realizaram os exames, tendo como principais anormalidades encontradas: elevação de PCR, Velocidade de Hemossedimentação (VHS), Anemia e Linfopenia, respectivamente; enquanto 14 pacientes não tinham alterações. Em contrapartida, somente 17 pacientes no grupo Controle realizaram exames, sendo os principais achados aumento de VHS, linfopenia e aumento de creatinina, respectivamente. Observamos diferença entre os grupos em relação ao aumento de PCR, mais comum no grupo Caso $(p=0,000047)$ e no aumento de creatinina, maior no grupo controle $(p=0,027)$.

\section{Avaliação dos pacientes do Grupo CASO com e sem Comorbidades}

Visando uma melhor compreensão do impacto das comorbidades nos pacientes com LES acometidos pelo SARS-COV-2 (pacientes CASO), esse quantitativo foi novamente dividido em dois grupos: Caso Com Comorbidades, com 53 pacientes(69,7\%); e Caso Sem Comorbidades com 23 pacientes(30,3\%).

A duração dos sintomas variou entre 1 a 20 ou mais dias, sendo que a maioria foi de até 5 dias, todavia, os pacientes com comorbidades associadas apresentaram um período sintomático mais prolongado (Gráfico 1). 
Gráfico 1 - Duração do período sintomático de COVID-19 nos pacientes com e sem comorbidades $(n=76)$ $60,00 \%$

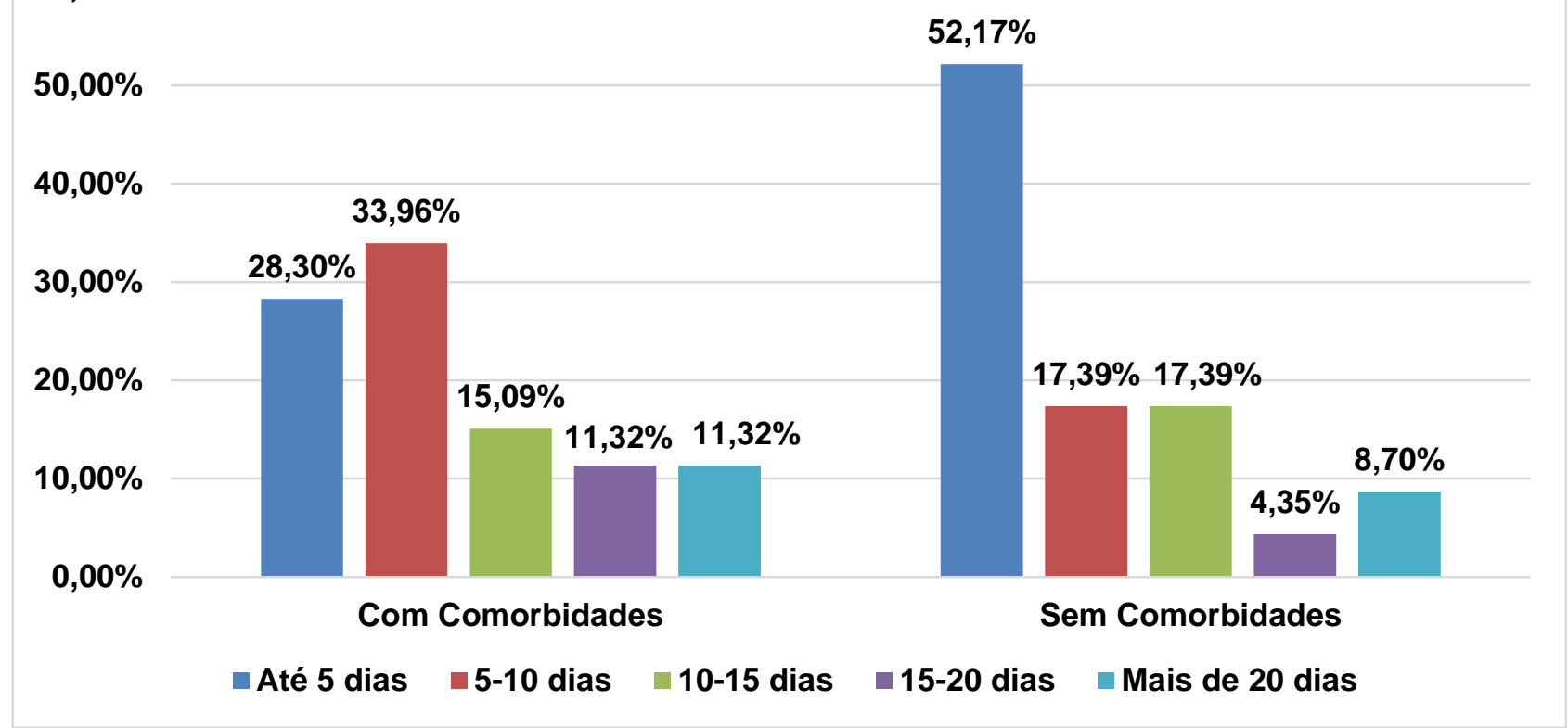

Fonte: Franco AM, et al., 2021.

Sobre a prevalência dos sintomas, não foram observadas diferenças significativas entre os grupos. Os principais sintomas foram: Astenia, Cefaleia, Coriza, Febre, Tosse, Perda de Olfato e Paladar e Dispneia, respectivamente (Tabela 3).

Tabela 3 - Prevalência dos Sintomas de COVID-19 nos 76 pacientes com e sem comorbidades

\begin{tabular}{|c|c|c|c|c|c|}
\hline \multirow{2}{*}{ Variável } & \multicolumn{2}{|c|}{$\begin{array}{c}\text { Com comorbidade } \\
N=53(69,7 \%)\end{array}$} & \multicolumn{2}{|c|}{$\begin{array}{c}\text { Sem comorbidade } \\
N=23(30,3 \%)\end{array}$} & \multirow{2}{*}{ P-valor } \\
\hline & $\begin{array}{l}\text { Frequência } \\
\text { absoluta (n) }\end{array}$ & $\begin{array}{l}\text { Frequência } \\
\text { relativa (\%) }\end{array}$ & $\begin{array}{l}\text { Frequência } \\
\text { absoluta (n) }\end{array}$ & $\begin{array}{l}\text { Frequência } \\
\text { relativa (\%) }\end{array}$ & \\
\hline Assintomático & 1 & $1,89 \%$ & 1 & $4,35 \%$ & 0,538 \\
\hline Alterações de pele & 5 & $9,43 \%$ & 3 & $13,04 \%$ & 0,638 \\
\hline Artralgias & 16 & $30,19 \%$ & 5 & $21,74 \%$ & 0,449 \\
\hline Astenia & 37 & $69,81 \%$ & 12 & $52,17 \%$ & 0,140 \\
\hline Cefaleia & 35 & $66,03 \%$ & 13 & $56,52 \%$ & 0,430 \\
\hline Coriza & 27 & $50,94 \%$ & 10 & $43,48 \%$ & 0,549 \\
\hline Diarreia & 9 & $16,98 \%$ & 6 & $26,09 \%$ & 0,360 \\
\hline Dispneia & 21 & $39,62 \%$ & 8 & $34,78 \%$ & 0,690 \\
\hline Febre & 22 & $41,5 \%$ & 14 & $60,87 \%$ & 0,120 \\
\hline Mialgias & 13 & $24,53 \%$ & 8 & $34,78 \%$ & 0,358 \\
\hline Náuseas & 10 & $18,87 \%$ & 4 & $17,39 \%$ & 0,878 \\
\hline Perda de olfato & 27 & $50,94 \%$ & 9 & $39,13 \%$ & 0,343 \\
\hline Perda de paladar & 25 & $47,17 \%$ & 9 & $39,13 \%$ & 0,517 \\
\hline Tonturas & 9 & $16,98 \%$ & 3 & $13,04 \%$ & 0,665 \\
\hline Tosse & 26 & $49,05 \%$ & 10 & $43,48 \%$ & 0,655 \\
\hline Vômitos & 3 & $5,66 \%$ & 2 & $8,70 \%$ & 0,624 \\
\hline Odinofagia & 9 & $16,98 \%$ & 8 & $34,78 \%$ & 0,087 \\
\hline
\end{tabular}

Fonte: Franco AM, et al., 2021.

A análise laboratorial dos 60 pacientes caso com exames disponíveis, constatou-se uma diferença significativa na maior incidência de aumento de VHS naqueles que tinham doenças associadas $(p=0,0045)$, além do percentual maior de aumento de VHS, DHL e aumento de creatinina nesse grupo, enquanto os participantes sem comorbidades tiveram maior percentual de exames normais, anemia, linfopenia e leucopenia (Gráfico 2). 
Gráfico 2 - Achados laboratoriais dos 45 pacientes do grupo Caso Com Comorbidades e 15 do grupo Caso Sem Comorbidades $(n=60)$.

\section{Caso com Comorbidade}

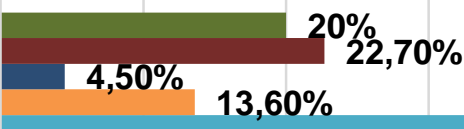

\section{Caso sem Comorbidade}
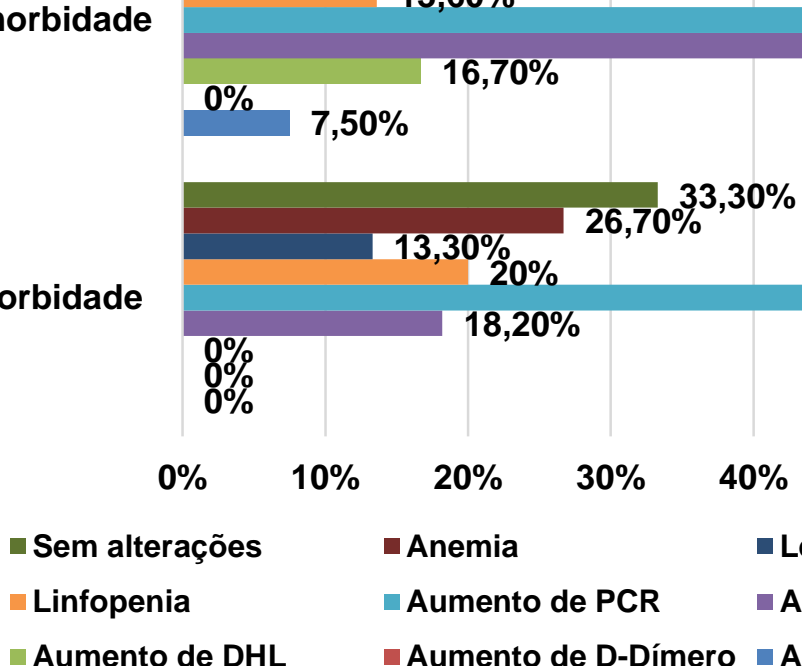

\section{$18,20 \%$}

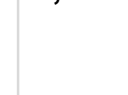

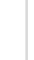

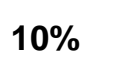

$20 \%$

$30 \%$

- Anemia

- Aumento de PCR

- Aumento de D-Dímero
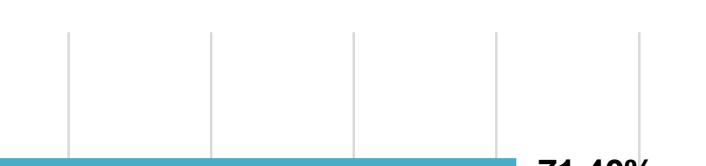

Fonte: Franco AM, et al., 2021.

Os desfechos clínicos dos pacientes com LES infectados pelo SARS-COV-2, no geral, foram de casos em que não houve necessidade de hospitalização, todavia a evolução com limitação de atividades, isto é, algum grau de dificuldade para realizar as atividades rotineiras, acometeu uma parcela importante desse grupo, sendo ainda mais expressivo nos indivíduos com doenças associadas. No estudo, apenas 4 pacientes foram hospitalizados, necessitando de $\mathrm{O}_{2}$ suplementar e 1 paciente foi a óbito em casa, sendo que todos pertenciam ao grupo Com comorbidades, apesar de não ter sido verificada diferença significativa entre os pacientes com e sem comorbidades estatisticamente $(p=0,241)$ (Gráfico 3).

Gráfico 3 - Desfechos clínicos dos pacientes com LES acometidos pela COVID-19.

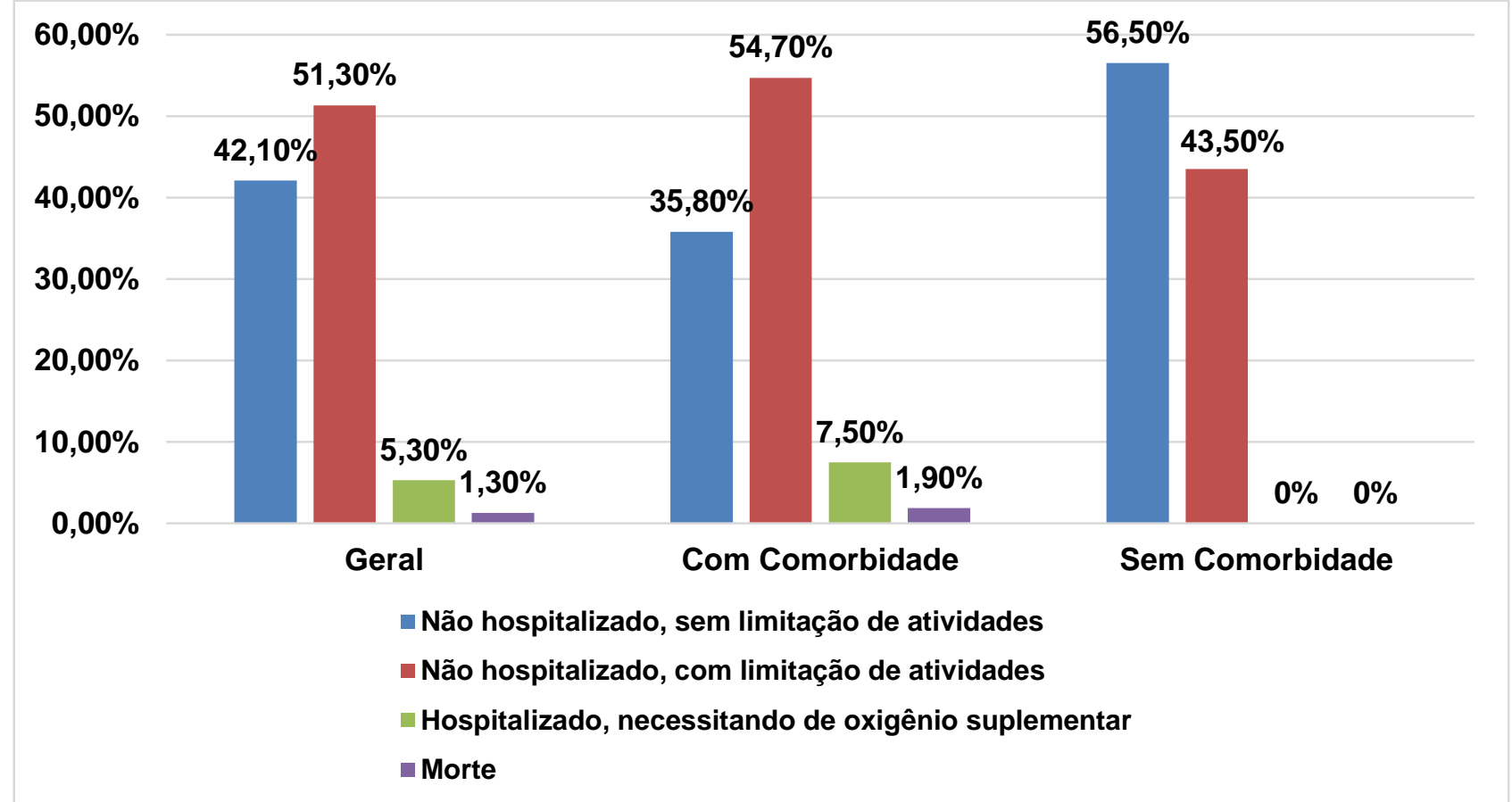

Fonte: Franco AM, et al., 2021. 


\section{DISCUSSÃO}

O presente estudo foi realizado com pacientes ambulatoriais portadores de LES com e sem COVID-19 na cidade de Manaus-Amazonas, com predomínio de indivíduos do sexo feminino, jovens e de cor parda. A análise das comparações entre o grupo Caso e Controle revelou não haver diferenças quanto à presença de comorbidades. Entretanto, encontramos maior incidência de aumento de marcadores inflamatórios, como o PCR. A presença de comorbidades cardiometabólicas foi reportada em mais da metade dos pacientes, sendo as mais comuns HAS e Obesidade. Não encontramos diferenças significativas entre os portadores de LES com e sem comorbidades cardiometabólicas, ainda que aqueles com comorbidades tenham apresentado período sintomático maior, marcadores inflamatórios elevados, maiores taxas de internação, evolução para óbito (1 paciente) e limitações de atividades pós-COVID-19 proporcionalmente.

A influência da simultaneidade da COVID-19 e outras doenças foi caracterizada negativamente por diversos autores, sendo apontadas como responsáveis por pior prognóstico e evolução da infecção, uma vez que o organismo já se encontrava fragilizado ou mesmo com condições favoráveis para replicação viral em maior escala, aproveitando-se de fatores fisiopatológicos das doenças de base. Ejaz $\mathrm{H}$, et al. (2020), avaliou que indivíduos com DM, doenças cardiovasculares (DCV), HAS, neoplasias e Síndrome da Imunodeficiência Adquirida (SIDA) tem maior risco de vida quando infectados pelo SARS-COV-2.

Fathi M, et al. (2020), por sua vez, em uma metanálise com 74855 pacientes encontrou que $47,01 \%$ dos participantes possuíam alguma comorbidade que impactou negativamente no curso da infecção pelo SARSCOV-2, sendo as principais a HAS(25,10\%), DM(13,48\%), DCV(18,25\%) e Doença Renal Crônica(3,27\%). Pathangey G, et al. (2021) observou que há forte evidência de risco elevado de quadros mais severos de COVID-19 em portadores de DCV, do aparelho respiratório, renais, diabetes, obesidade e hipertensão. Yang $\mathrm{J}$, et al. (2020), também em uma metanálise com 1576 pacientes, relatou pacientes com COVID-19 grave tiveram maior prevalência de comorbidades do que os não graves.

A HAS foi uma das comorbidades com maior prevalência, sendo incluída na categoria de DCV que predispõem a complicações da infecção pelo SARS-CoV-2 (FERRARI F, et al. 2020). Fang X, et al. (2020), em uma metanálise com mais de 10.000 pacientes que a observou que HAS, assim como outras comorbidades, está associada com maior probabilidade de ocorrência de todos os seguintes desfechos: morte, evolução para SRAG, necessidade de suplementação de oxigênio por meio de via aérea avançada, admissão em UTI, disfunções cardíacas e progressão da doença.

A explicação para essa correlação entre a HAS e severidade do COVID-19, relaciona-se com o fato do vírus utilizar o receptor da ECA 2 como meio de infectar as células, levantando a hipótese de que o uso de anti-hipertensivos como os Inibidores da Enzima Conversora de Angiotensina (IECA) e Bloqueadores de Receptores de Angiotensina (BRA) poderiam sofrer uma regulação negativa e aumentar a expressão desses receptores, resultando em mais portas de entrada para o vírus (ZHENG YY, et al. 2020). Além disso, existe também a hipótese de que o dano miocárdico relacionado ao SARS-CoV-2 envolve a tempestade de citocinas e a quantidade excessiva de cálcio no interior das células, como resultado de um processo de apoptose dos cardiomiócitos em hipóxia (ASKIN L, et al. 2020).

Por outro lado, a Obesidade é relatada como fator de risco importante para a infecção pelo SARS-CoV-2, predispondo também a outras desordens metabólicas, além de apresentar um quadro pró-inflamatório préexistente que pode agravar o curso da COVID-19 (SAMUELS JD, 2020).

Palaiodimos L, et al. 2020, acompanhou 200 pacientes com COVID-19 internados em Nova lorque:24\% faleceram durante a internação, sendo que desses apenas 31,6\% tinham IMC normal, enquanto 17,2\% tinham sobrepeso, obesidade grau I ou II e $34,8 \%$ obesidade grau III. A obesidade foi considerada como um fator de risco independente para maior mortalidade e necessidade de cuidados intensivos. Nakeshbandi M, et al., 2020, avaliou 504 pacientes com COVID-19 e os dividiu em peso normal, com 139 pacientes(27\%); sobrepeso, com 150 pacientes(30\%); e obesidade, com 215 pacientes(43\%). Ao final do estudo, constatouse risco maior de morte e necessidade de ventilação mecânica nos grupos sobrepeso e obesidade em comparação com o peso normal. 
A expressão do receptor de ECA 2 abundante no tecido adiposo, assim como nos pulmões, torna os pacientes obesos mais vulneráveis (PANDEY A, et al. 2018). Sabe-se também que alterações endoteliais podem estar presentes na evolução da COVID-19, culminando em distúrbios de coagulação, e a obesidade por si só também está associada a disfunção endotelial (VARGA Z, et al. 2020). Os obesos possuem ainda um comprometimento da função respiratória, com diminuição de capacidade funcional, volume de reserva expiratório e complacência (DIETZ W e SANTOS-BURGOA C, 2020). Ter em mente os mecanismos fisiopatológicos da COVID-19 associada a comorbidades como a HAS e a Obesidade é fundamental para orientar o manejo adequado de pacientes potencialmente mais graves, sejam eles portadores de doenças reumáticas, como o LES, ou não.

Na literatura, alguns estudos apontam a elevação de marcadores inflamatórios nos pacientes com COVID19: Cunha DBA, et al. (2021), verificou que o PCR, assim como outros exames que avaliam perfil inflamatório, é considerado um exame indicativo de gravidade da COVID-19. Tjendra Y, et al. (2020), afirma que apesar de os marcadores inflamatórios de fase aguda como PCR, ferritina, procalcitonina e amiloide A sérico serem considerados inespecíficos, eles são sensíveis à COVID-19 e os respectivos aumentos estão associados com alta incidência de complicações da infecção. Hariyanto TI, et al. (2021), em uma metanálise com 4848 pacientes, encontrou níveis aumentados de procalcitonina, PCR, D-Dímero e DHL em pacientes com COVID19 grave, sendo esses exames considerados úteis para predizer quadros graves da doença.

Em nosso estudo, a influência das comorbidades no curso clínico dos pacientes com LES e COVID-19 foi demonstrada, porém sem grande relevância, como era esperado. De acordo com o que foi descrito por outros autores: Emmi G, et al. (2020) avaliou a prevalência de infecção pelo SARS-CoV-2 em 458 portadores de doenças reumáticas, 13 desenvolveram COVID-19, 4 com LES e 1 Síndrome de Sjogren que necessitaram de tratamento de suporte avançado. E 39\% estavam em atividade da doença. Comparando a prevalência de casos de COVID-19 na população em geral(0,20\%) e do grupo estudado(0,22\%), a diferença não foi significativa.

Favalli EG, et al. (2020), acompanhou 900 pacientes com LES, onde 8 com sintomas de síndrome gripal, sem necessidade de internação e 3 tiveram contato com casos confirmados de COVID-19, porém assintomáticos. Isso revelou um baixo impacto da doença nos portadores de LES. Ahmed S, et al. (2021), reiterou que pacientes com doenças reumáticas parecem não ter um risco aumentado de severidade do COVID-19, seja por conta da doença de base ou do tratamento imunossupressor. Todavia, é comum encontrar nesses pacientes muitas das doenças que são consideradas fatores de risco, então é necessário realizar um acompanhamento rigoroso.

Santos CS, et al. (2020), avaliou 3711 pacientes internados com COVID-19, sendo 38(10\%) com doenças reumáticas. Dentre eles, $26 \%$ morreram, e os principais fatores de risco associados a esse acontecimento foram, respectivamente: atividade da doença reumática, dislipidemia, DCV e doenças respiratórias. Havendo também impacto significativo de HAS e DM. Apesar disso, a maioria dos pacientes evoluiu com cura da infecção. Esses achados, destoam de hipóteses levantadas inicialmente de que pacientes com LES e comorbidades teriam pior prognóstico, seja por conta da doença de base ou das práticas terapêuticas com imunossupressores utilizadas (CERIBELLI A, et al. 2020).

\section{CONCLUSÃO}

A frequência de comorbidades cardiometabólicas nos pacientes com LES dos grupos caso e controle foi semelhante, destacando-se somente a maior prevalência de HAS no grupo controle. A influência dessas doenças nos pacientes com LES sobre a curso da COVID-19 foi pequena, visto que as maiores prevalências de período sintomático prolongado, marcadores inflamatórios, hospitalizações, óbitos e limitação de atividades após a cura da infecção não tinham relevância estatística, todavia não podem ser desconsiderados na avaliação e manejo clínico desses pacientes. Os resultados deste estudo podem refletir uma possível ação do uso imunossupressores no tratamento das doenças reumáticas no controle da resposta inflamatória verificada nos casos mais graves de COVID-19. Contudo, mais estudos são necessários para validar essa hipótese. 


\section{AGRADECIMENTOS E FINANCIAMENTO}

Agradecemos ao Serviço de Reumatologia do AAL, ao programa de iniciação científica da UFAM, ao $\mathrm{CNPq}$, e a todos os profissionais que contribuíram na execução deste estudo. Assim como, agradecemos a todos que se dispuseram a participar da pesquisa, aos familiares e amigos que contribuíram grandemente para que fosse possível realizar a pesquisa.

\section{REFERÊNCIAS}

1. AHMED S, et al. Comorbidities in rheumatic diseases need special consideration during the COVID-19 pandemic. Rheumatology International, 2021; 1-14.

2. ASKIN L, et al. O Efeito da Doença de Coronavírus 2019 nas Doenças Cardiovasculares. Arquivos Brasileiros de Cardiologia, 2020; 817-822.

3. BAI Y, et al. Presumed Asymptomatic Carrier Transmission of COVID-19. JAMA - Journal of the American Medical Association, 2020; 1406-1407.

4. CERIBELLI A, et al. Recommendations for coronavirus infection in rheumatic diseases treated with biologic therapy. Journal of Autoimmunity, 2020;102442.

5. CUNHA DBA, et al. Uso de marcadores biológicos para avaliação prognóstica de pacientes com covid-19: uma revisão de literatura. RECIMA21 - Revista Científica Multidisciplinar, 2021;2675-6218.

6. DIETZ W, SANTOS-BURGOA, C. Obesity and its Implications for COVID-19 Mortality. Obesity - A Research Journal, $2020 ; 22818$.

7. EJAZ H, et al. COVID-19 and comorbidities: Deleterious impact on infected patients. Journal of Infection and Public Health, 2020; 1833-1839.

8. EMMI G, et al. SARS-CoV-2 infection among patients with systemic autoimmune diseases. In Autoimmunity Reviews, 2020; 102575.

9. FANG X, et al. Epidemiological, comorbidity factors with severity and prognosis of COVID-19: a systematic review and meta-analysis. Aging (Albany NY) 12.13, 2020; 12493.

10. FATHI M, et a. Prognostic value of comormidity for severity of covid-19: a systematic review and meta-analysis study. MedRxiv,2020.

11. FAVALLI EG, et al. Are patients with systemic lupus erythematosus at increased risk for COVID-19? In Annals of the Rheumatic Diseases. BMJ Publishing Group, 2020; 217787.

12. FERRARI F, et al. Ponto de Vista COVID-19: Dados Atualizados e sua Relação Com o Sistema Cardiovascular. Arquivos Brasileiros de Cardiologia, 2020; 823-826.

13. HARIYANTO TI, et al. Inflammatory and hematologic markers as predictors of severe outcomes in COVID-19 infection: A systematic review and meta-analysis. The American journal of emergency medicine 2020; 110-119.

14. JOHNS HOPKINS CORONAVIRUS RESOURCE CENTER (JHCRC). COVID-19 MAP - Disponível em: https://coronavirus.jhu.edu/map.html. Acesso em 16 de Agosto de 2021.

15. KENJI O, TATSUYA A. Systemic Lupus Erythematosus: nothing stale her infinite variety. Modern Rheumatology, 2018; 758-765.

16. MEHTA P, et al. COVID-19: consider cytokine storm syndromes and immunosuppression. In The Lancet, 2020; 10331034.

17. MINISTÉRIO DA SAÚDE. Rede Nacional de Dados em Saúde (RNDS). COVID-19 Vacinação. Disponível em: Https://Qsprod.Saude.Gov.Br/Extensions/DEMAS_C19Vacina/DEMAS_C19Vacina.Html. Acesso em: 16 de agosto de 2021

18. MUNIYAPPA R, GUBBI S. COVID-19 pandemic, coronaviruses, and diabetes mellitus. American Journal of Physiology, 2020; 736-741.

19. NAKESHBANDI M, et al. The impact of obesity on COVID-19 complications: a retrospective cohort study. International journal of obesity, 2020; 44(9): 1832-1837.

20. O'NEILL S, CERVERA R. Systemic lupus erythematosus. Best Practice \& Research Clinical Rheumatology, 2010; 24(6): 841-855.

21. PALAIODIMOS L, et al. Severe obesity, increasing age and male sex are independently associated with worse inhospital outcomes, and higher in-hospital mortality, in a cohort of patients with COVID-19 in the Bronx, New York. Metabolism: Clinical and Experimental, 2020; 108: 154262.

22. PANDEY A, et al. Physical Activity, Fitness, and Obesity in Heart Failure With Preserved Ejection Fraction. In JACC: Heart Failure, 2018; 975-982.

23. PATHANGEY G, et al. Angiotensin-converting enzyme 2 and COVID-19: patients, comorbidities, and therapies.American Journal of Physiology-Lung Cellular and Molecular Physiology 320.3 (2021): L301-L330. 
24. PETRI M, et al. Derivation and Validation of Systemic Lupus International Collaborating Clinics Classification Criteria for Systemic Lupus Erythematosus. Arthritis Rheum, 2012; 64(8): 2677-2686.

25. ROSER H. Coronavirus (COVID-19) Vaccinations - Statistics and Research - Our World in Data. Disponível em: Https://Ourworldindata.Org/Covid-Vaccinations?Country=OWID_WRL. Acesso em: 16 de agosto de 2021.

26. ROTHAN HA, BYRAREDDY SN. The epidemiology and pathogenesis of coronavirus disease ( COVID-19) outbreak. Journal of Autoimmunity, February, 2020; 102433.

27. SAMUELS JD. Obesity and severe COVID-19 disease: a strong association. Obesity - A Research Journal, 2020: 02.

28. SANTOS CS, et al. Determinants of COVID-19 disease severity in patients with underlying rheumatic disease. Clinical Rheumatology, 2020; 2789-2796.

29. TJENDRA Y,et al. Predicting disease severity and outcome in COVID-19 patients: a review of multiple biomarkers. Archives of pathology \& laboratory medicine, 2020; 144(12): 1465-1474.

30. VARGA Z, et al. Endothelial cell infection and endotheliitis in COVID-19. In The Lancet, 2020; 1417-1418.

31. YANG J, et al. Prevalence of comorbidities and its effects in patients infected with SARS-CoV-2: a systematic review and meta-analysis. International Journal of Infectious Diseases, 2020; 94: 91-95.

32. ZHENG YY, et al. COVID-19 and the cardiovascular system. In Nature Reviews Cardiology, 2020; 259-260.

33. ZHOU F, et al. Clinical course and risk factors for mortality of adult inpatients with COVID-19 in Wuhan, China: a retrospective cohort study. The lancet. 2020; 395(10229): 1054-1062.

34. ZHU N, et al. A novel coronavirus from patients with pneumonia in China, 2019. New England Journal of Medicine, 2020; 382(8): 727-733. 